\title{
Speech Perception through Face Masks by Children and Adults
}

Julia Schwarz¹ (js2275@cam.ac.uk), Katrina Kechun Li1 (k1502@cam.ac.uk),

Jasper Hong Sim ${ }^{1}$, Yixin Zhang ${ }^{1}$, Elizabeth Buchanan-Worster ${ }^{2}$, Brechtje Post ${ }^{1}$, Jenny Gibson ${ }^{3}$, Kirsty McDougall ${ }^{1}$

1) Theoretical and Applied Linguistics 2) MRC CBU 3) Faculty of Education

\section{Introduction}

People report that face masks can cause communication difficulties. We investigated aspects of speech processing which might cause these difficulties.

\section{- Face masks create a visual barrier} Visual cues from the speaker's face and mouth can improve speech intelligibility significantly, even when the image is imperfect (e.g. due to distance in classrooms).

\section{- Face masks cause acoustic changes}

For example, masks can reduce the overall intensity and cause changes to high frequencies. However,

there is limited evidence that these acoustic changes affect speech recognition accuracy. Mild processing cost may be captured more effectively measuring reaction times (RT) instead of effectively

- Semantic sentence predictions are vital to processing connected speech.

This is especially important when the signal is distorted. High predictability could reduce visual and auditory processing difficulties caused by face masks.

- Children are less experienced in using semantic cues for predictive speech processing.

- A concern might be that children cannot use semantic cues as effectively as adults to

II compensate for adverse listening conditions. Mask research has so far concentrated on adults.

\section{Research Questions}

1. Does listening to speech through face masks increase mental effort, affecting processing speed (RT)?

2. What are the relative contributions from visual blocking vs. acoustic changes?

3. Can linguistic context (semantic predictability) make up for (acoustic) bottom-up processing difficulties?

4. Do the three effects (visual, acoustic, predictability) affect children in the same way as adults?

\section{Methods \& Materials}

Participants (preliminary subsample)

16 British English (BE) adults (age 20-60), 16 BE children (age 8-12)

\section{Materials}

120 target words embedded in sentence-final position, manipulated by Visual Mask Effect $\times$ Acoustic Mask Effect $\times$ Semantic Predictability of the target word (cloze probability)

Examples: For your birthday I baked this cake. (High) Tom wants to know about this cake. (Low)

\begin{tabular}{|c|c|c|c|}
\hline & & (4) Visual No Mask & Visual Mask $\_$ \\
\hline $\begin{array}{l}\text { Acoustic } \\
\text { No Mask }\end{array}$ & 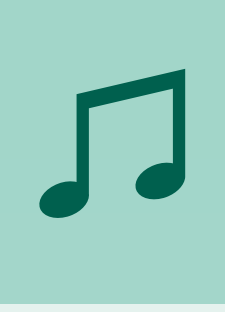 & $\begin{array}{l}\text { High vs. Low } \\
\text { Target Predictability }\end{array}$ & $\begin{array}{l}\text { High vs. Low } \\
\text { Target Predictability }\end{array}$ \\
\hline $\begin{array}{l}\text { Acoustic } \\
\text { Mask }\end{array}$ & Ґ] & $\begin{array}{l}\text { High vs. Low } \\
\text { Target Predictability }\end{array}$ & $\begin{array}{l}\text { High vs. Low } \\
\text { Target Predictability }\end{array}$ \\
\hline
\end{tabular}

Procedure

- Experiment conducted online on Gorilla experiment builder

- Participants watched videos of a BE speaker and repeated the last word of the sentence that they heard (cued shadowing).

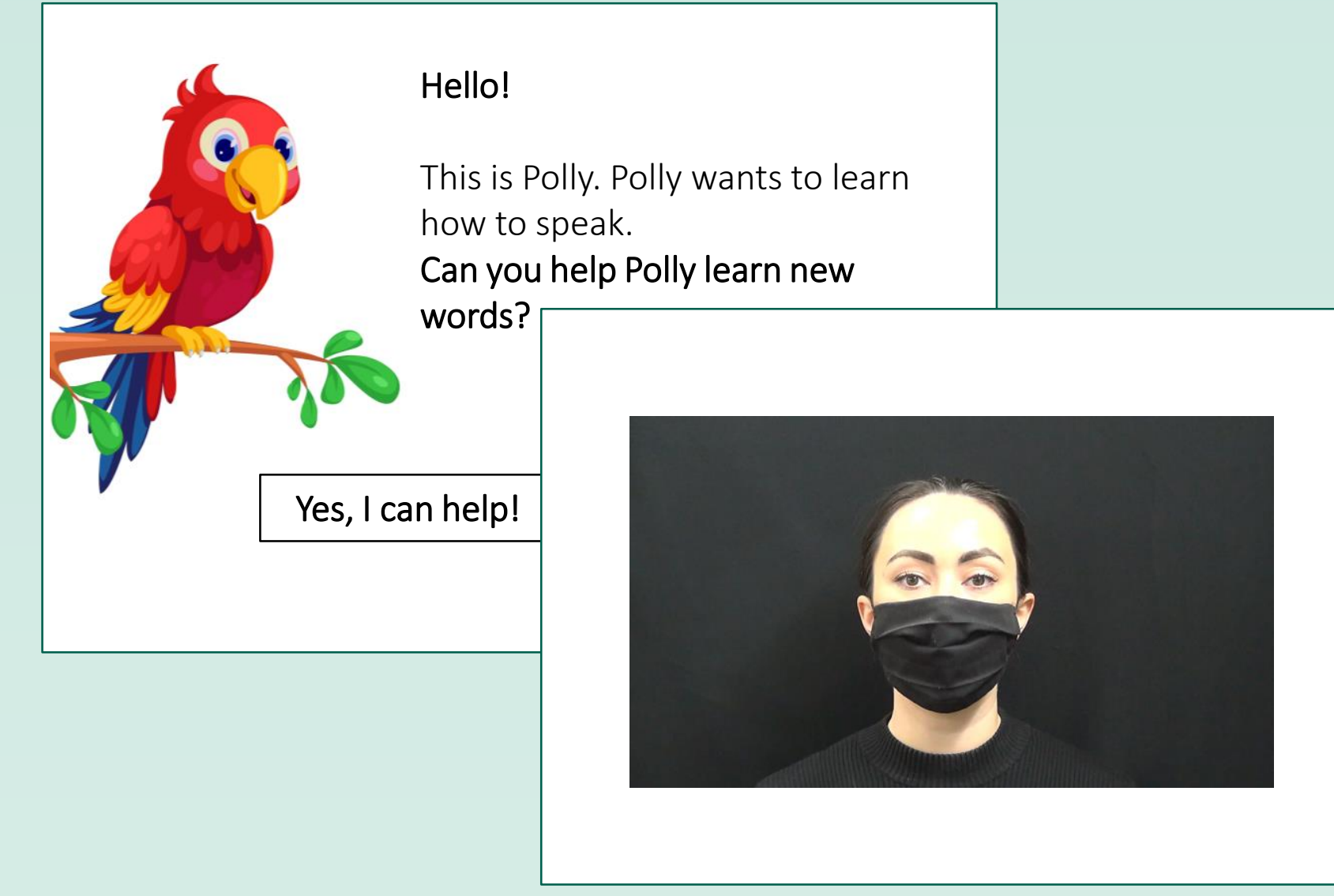

\section{Analysis}

- A sound signal at the beginning of each sentence serves as an anchor for calculating reaction times.

- With this new technique, sound signal $x$-[sentence duration] = RT cued shadowing can be conducted online with high precision and accuracy, with children and adults.



- Participant responses are marked with the help of automatic speech onset recognition (Chronset) and manual corrections.

- Reaction times are statistically analysed with mixed effects modelling with four main effects (visual cues, acoustic cues, predictability, age group) and respective two-way interactions.

\section{Results (preliminary subsample)}
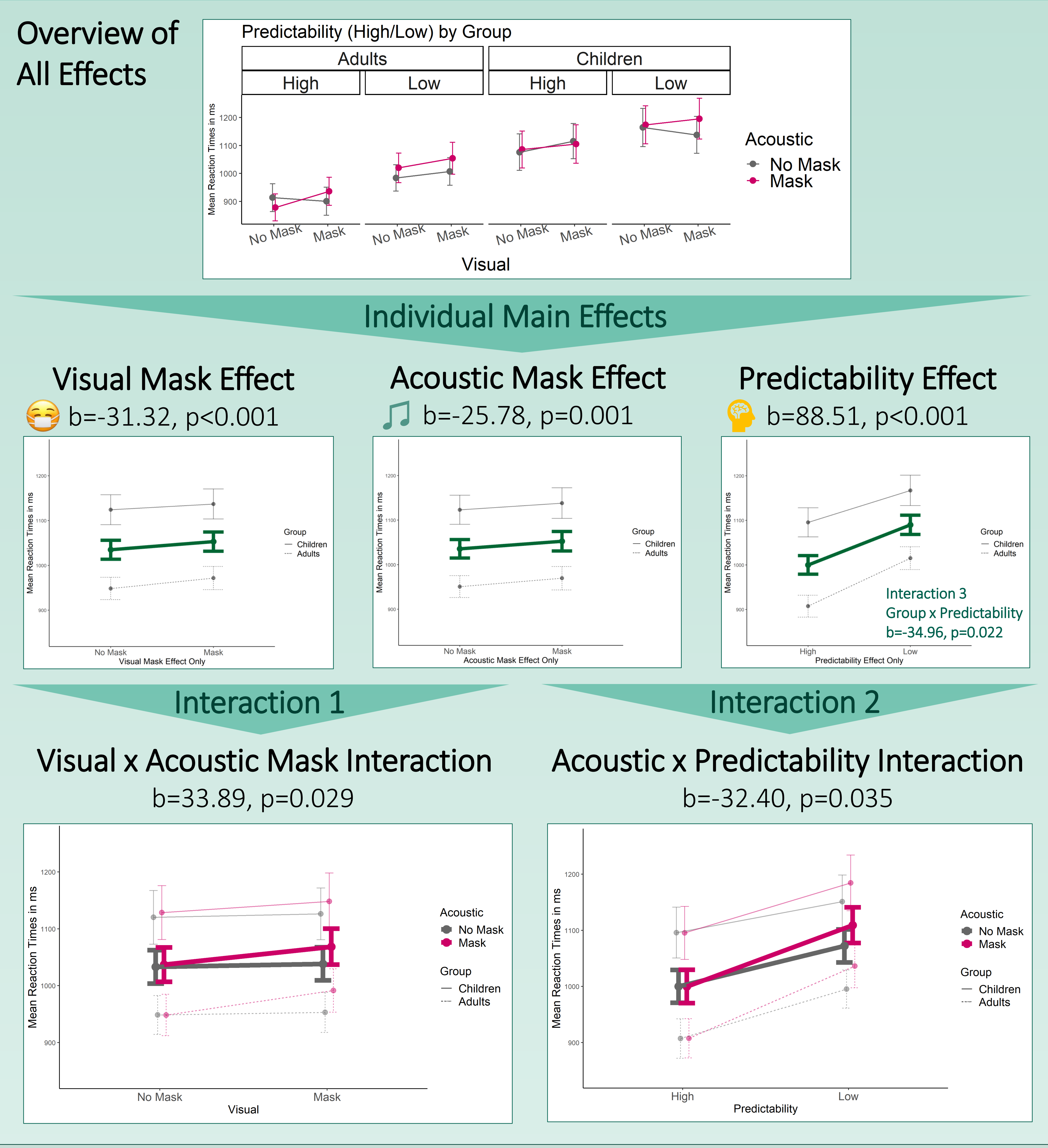

\section{Conclusions}

1. Listening to speech through face masks slows down processing, leading to longer response latencies.

2. Visual and acoustic mask effects individually are very small (10ms), but interestingly show a larger effect in combination (40ms) when one modality cannot compensate for the other.

3. High semantic predictability reduces these mask effects significantly overall, and largely makes up for acoustic processing difficulties.

4. Children are less proficient in predictive processing, but use semantic cues to compensate for mask effects in a similar fashion to adults.

Acknowledgements: Many thanks to Lorna Halliday, Hugo Caffaratti, and Alice Paver for their help with this project. This project was possible thanks to the Cambridge Language Sciences Incubator Fund.

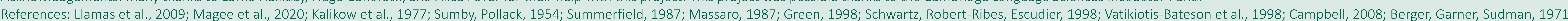

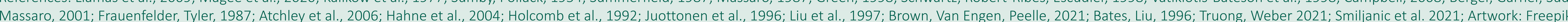

